\title{
New Insulins and Insulin Therapy
}

\author{
Thomas Danne ${ }^{1}$ and Jan Bolinder ${ }^{2}$
}

\section{Introduction}

$\mathrm{T}$ HE RECENT PUBLICATION of the ORIGIN Trial offered assurance to the area of insulin and insulin analogs in diabetes. This trial of 12,537 people did not support epidemiologic analyses that have linked insulin in general, or insulin glargine in particular, to incident cancers during several years of exposure. Such detailed studies into the safety and efficacy are called for, not only for new insulin but also for biosimilar insulins, as it is very likely that in the next years these will come to the market, also in highly regulated markets. Differences in the manufacturing process of biosimilars may result in differences from the originator insulin to some extent. Currently we are still waiting for data on these not so new "new insulins."

Compared with the physiology of basal insulin in subjects without diabetes, the current long-acting insulin analogs still present limitations. As it appears difficult to show improvements in HbA1c simply by comparing two different insulin formulations, the variability of effects on blood glucose control with subsequent reduction in hypoglycemia has become the focus of recent trials. More and more insulin studies use continuous glucose monitoring as an unambiguous tool to look at glucose variability and frequency and duration of hypoglycemic episodes compared to previous efforts relying on logbooks and self blood glucose monitoring.

In the past year, insulin detemir has received the pediatric indication as the pivotal phase III trial was completed. Initial phase III studies of the ultra long-acting insulin degludec have been published also, where the efficacy and safety of this basal insulin analog have been compared with insulin glargine in patients with type 1 and type 2 diabetes. Eli Lilly (Indianapolis, Indiana) has described a long-acting lispro insulin formulation that is PEGylated. Initial data that is available only online indicates that PEGylated lispro in normal humans without diabetes has a flatter action profile compared with insulin glargine along with a prolonged activity.

With regard to short-acting insulins for mealtime insulin supplementation, the controversy continues regarding the three available rapid-acting analogs-lispro, aspart, and glulisine-in CSII therapy (1). A closer look into the data revealed that there may be relevant differences between them that warrant further study (2). The search for a new insulin with a more rapid onset of action continues. Last year we reported on regulatory delays for Linjeta ${ }^{\mathrm{TM}}$ (formerly named VIAject), a human insulin formulation with faster insulin absorption and action than insulin lispro and regular human insulin. The news of a National Institutes of Health (NIH) grant for the development of concentrated ultra rapid-acting insulin formulations for use in an artificial pancreas to Linjetadeveloper Biodel promises more data next year. All in all, this review demonstrates that also in 2012 significant advances in our understanding of new insulins have been made. As we comment on the recent trials, we are looking forward to the results of large clinical trials such as ORIGIN to tell us if the potential advantages of new insulins eventually lead to meaningful improvements in the everyday management of patients with diabetes.

\section{LONG-ACTING INSULIN ANALOGS: INSULIN GLARGINE-NO LINK TO CANCER FOUND}

\section{Basal insulin and cardiovascular and other outcomes in dysglycemia}

The ORIGIN Trial Investigators

N Engl J Med 2012; 367: 319-328

\section{Background}

The primary aim of this trial was to test if provision of sufficient basal insulin to normalize fasting plasma glucose levels may reduce cardiovascular events, but the scope and duration of the study allowed also to give meaningful data regarding a potential link between insulin and cancer.

\section{Methods}

A group of 12,537 people (mean age, 63.5 years) with cardiovascular risk factors plus impaired fasting glucose, impaired glucose tolerance, or type 2 diabetes were randomly assigned to receive insulin glargine (with a target fasting blood glucose level of $\leq 95 \mathrm{mg}$ per deciliter [ $5.3 \mathrm{mmol}$ per

\footnotetext{
${ }^{1}$ Diabetes-Zentrum für Kinder and Jugendliche, Kinder- und Jugendkrankenhaus AUF DER BULT, Hannover, Germany
}

${ }^{2}$ Department of Medicine, Karolinska University Hospital Huddinge, Karolinska Institutet, Stockholm, Sweden 
liter]) or standard care, and to receive $\mathrm{n}-3$ fatty acids or placebo with the use of a 2-by-2 factorial design.

\section{Results}

No significant difference between insulin glargine and standard care was found in each component of the two coprimary outcomes or in the incidence of any cancer (hazard ratio, 1.00; $95 \% \mathrm{CI}, 0.88$ to $1.13 ; \mathrm{p}=0.97$ ), death from cancer (hazard ratio, $0.94 ; 95 \% \mathrm{CI}, 0.77$ to $1.15 ; \mathrm{p}=0.52)$, or cancer at specific sites.

\section{Conclusions}

When used to target normal fasting plasma glucose levels for more than 6 years, insulin glargine had a neutral effect on cardiovascular outcomes and cancers. Although it reduced new-onset diabetes, insulin glargine also increased hypoglycemia and modestly increased weight.

\section{Comment}

Although this yearbook focuses on type 1 diabetes, this type 2 article is of special interest regarding the discussion of a potential mitogenicity of insulin analogues, particularly of insulin glargine. We have reviewed the controversial, circumstantial, epidemiological evidence about the link between insulin and cancer in previous years. This is now the longest prospective study in a large number of individuals receiving insulin glargine. Although it was not the primary objective of the study, and the cancer issue came up after the ORIGIN Trial was already in progress, it is the best scientific data we have so far to answer the question. And the comforting result in the words of the ORIGIN researchers follows: "There was not an increase in incident cancers; thus, these data do not support epidemiologic analyses that have linked insulin in general or insulin glargine in particular to incident cancers during several years of exposure." (8)

\section{Biosimilars-Not There Yet}

Until now, most insulin is manufactured and distributed by a small number of multinational companies. However, other pharmaceutical companies-many of these located in countries such as India or China-are also able to manufacture insulin with modern biotechnological methods. This becomes particularly relevant as the patents for many insulin formulations have expired or are going to expire soon. Thus, market approval may be applied for with such so-called "biosimilar insulins," including in the EU or U.S. markets. Lutz Heinemann's recent review discusses the complexity of biosimilar insulin's approval and usage, as well as scientific and regulatory aspects (3). Although these insulins appear to be similar to approved insulin formulations ("biosimilar"), differences in the structure of the insulin molecule and/or the purity may have clinically relevant consequences for the biological effects. The guidelines already in place in the EU for market approval require that the manufacturer demonstrates that his biosimilar insulin has a safety and efficacy profile that is similar to that of the "original" insulin formulation. Recently, guidelines for biosimilars were issued in the United States; however, these do not cover insulin. Thus, the potential safety and efficacy issues surrounding biosimilar insulins are substantial and relevant. So far there is little published literature on these biosimilar compounds, and we will continue to monitor-as with any other new insulin-if a careful and evidence-driven approval process is initiated. Nevertheless, it is very likely that in the next few years, biosimilar insulin will become available on a larger scale.

\section{INSULIN DETEMIR: SAFE IN KIDS}

\section{Treatment with insulin detemir or NPH insulin in children aged 2-5yr with type 1 diabetes mellitus}

Thalange $N^{1}$, Bereket $A^{2}$, Larsen $J^{3}$, Hiort $L C^{4}$, Peterkova $V^{5}$

${ }^{1} J e n n y$ Lind Children's Department, Norfolk E Norwich University Hospital, Norwich, United Kingdom; ${ }^{2}$ Department of Paediatrics, Division of Paediatric Endocrinology and Diabetes, Marmara University School of Medicine, Istanbul, Turkey; ${ }^{3}$ Medical $\mathcal{E}$ Science, NovoNordisk A/S, Bagsvaerd, Denmark; ${ }^{4}$ Biostatistics Insulin \& Devices, NovoNordisk A/S, Bagsvaerd, Denmark; and ${ }^{5}$ Endocrinological Scientific Centre, Institute of Paediatric Endocrinology, Russian Academy of Science, Moscow, Russia

Pediatr Diabetes 2011; 12: 632-41

\section{Background}

This trial was necessary to get the pediatric indication for Levemir ${ }^{\circledR}$. It was a randomized (1:1), multinational, openlabeled parallel group trial comparing insulin detemir (IDet) with neutral protamine Hagedorn (NPH) insulin, in combination with mealtime insulin aspart, over one year in subjects aged 2-16 years with type 1 diabetes mellitus. Of 348 randomized subjects, 82 (23.6\%) were aged 2-5 years (IDet: 42 , NPH: 40). This article is a descriptive subgroup analysis of these young children.

\section{Methods}

Baseline characteristics (IDet vs. NPH) were similar: mean age, 4.3 vs. 4.5 years; diabetes duration, 2.2 vs. 2.1 years; males, 42.9 vs. $52.5 \%$.

\section{Results}

Mean hemoglobin A1c (HbA1c) was similar between groups at baseline (8.2 vs. $8.1 \%$ ) and changed little over one year $(8.1$ vs. $8.3 \%)$. Fasting plasma glucose (FPG) was similar at baseline ( 8.44 vs. $8.56 \mathrm{mmol} / \mathrm{L})$ and decreased during the study ( -1.0 vs. $-0.45 \mathrm{mmol} / \mathrm{L})$. A lower rate of hypoglycemia was observed with IDet compared with NPH (24-h; 50.6 vs. 78.3 episodes per patient-year; nocturnal hypoglycemia, 8.0 vs. 17.4 episodes per patient-year). No severe hypoglycemic episodes occurred with IDet, while three subjects reported six episodes with NPH. Change in weight standard deviation score standardized by age and gender was -0.17 with IDet and +0.03 with NPH. A slightly lower proportion of subjects in this age group reported adverse events with IDet than with $\mathrm{NPH}(69.0$ vs. $77.5 \%$ ). Serious adverse events were few (5 with IDet, 7 with NPH).

\section{Conclusions}

In conclusion, long-term treatment with IDet in children aged $2-5$ years suggested similar glycemic control, greater 
reduction in FPG, lower rates of hypoglycemia, no inappropriate weight gain, and fewer adverse events compared with $\mathrm{NPH}$.

\section{Comment}

Although the full study results including subjects 6 to 16 years of age are only published in abstract form so far, analyses in both age groups confirm previous observations regarding the efficacy and safety in pediatric and adult studies. Although insulin detemir does not appear to be superior to NPH regarding average glycemic control, as judged by $\mathrm{HbA1c}$, it is of note that less (nocturnal) hypoglycemia is observed with this long-acting analogue compared to NPH. In addition, it is the only basal insulin in which no untoward weight gain was observed. This favorable effect of insulin detemir has been described in several studies and is still poorly understood. Meanwhile, use of insulin detemir is a well-established practice in pediatric patients.

\section{HEAD-TO-HEAD COMPARISON BETWEEN INSULIN DETEMIR AND INSULIN GLARGINE IN TYPE 2 DIABETES}

\section{Insulin detemir versus insulin glargine for type 2 diabetes mellitus}

Swinnen SG, Simon ACR, Holleman F, Hoekstra JB, DeVries JH

Internal Medicine, Academic Medical Centre, Amsterdam, The Netherlands

Cochrane Database Syst Rev 2011; 7: CD006383

\section{Background}

To directly compare the effects of insulin detemir and insulin glargine in patients with type 2 diabetes mellitus.

\section{Methods}

Meta-analysis of all randomized, controlled trials in patients with type 2 diabetes comparing the effects of insulin detemir and insulin glargine, with a study duration of at least 12 weeks. Pooling of the studies by means of random-effects meta-analysis was performed.

\section{Results}

Four trials lasting between 24 to 52 weeks and comprising a total of 2,250 patients with type 2 diabetes mellitus randomized to insulin detemir or insulin glargine were included in the meta-analysis. Initiation of once-daily (evening time) injection of insulin detemir with the possibility to add an additional dose in the morning was devised in three studies, whereas in the remaining study, insulin detemir therapy was given twice daily from the start. Altogether, $13.6 \%$ to $57.2 \%$ of the patients allocated to insulin detemir in the various trials were on twicedaily injections at the end of the study period. Insulin glargine was given once daily (evening time) in all studies. Glycemic control, assessed as $\mathrm{HbA} 1 \mathrm{c}$ and $\mathrm{HbA} 1 \mathrm{c} \leq 7 \%$ with or without hypoglycemic events, was comparable in the treatment groups, and there were no statistically significant differences in overall, nocturnal, or severe hypoglycemia. Neither were there any significant differences in the variability of fasting plasma glucose or glucose levels in 24-hour profiles. Treatment with insulin detemir resulted in significantly less weight gain, whereas insulin glargine was associated with lower daily basal insulin dose and less injection site reactions. It was not possible to draw any conclusions regarding quality of life, costs, or mortality.

\section{Conclusions}

There is no clinically relevant difference in efficacy or safety between insulin detemir and insulin glargine in patients with type 2 diabetes. However, to achieve the same level of glycemic control, insulin detemir treatment often necessitated twice-daily injections with a higher daily dose but with less weight gain, whereas insulin glargine was given once daily and with fewer injection site reactions.

\section{Comment}

Most studies evaluating the efficacy and safety of insulin detemir and insulin glargine in patients with diabetes mellitus have been performed with NPH-insulin as comparator, and, overall, direct head-to-head comparisons between the two long-acting basal insulin analogs are scarce. This meta-analysis summarizes the data from four randomized controlled trials directly comparing basal insulin supplementation with insulin detemir and insulin glargine in patients with type 2 diabetes mellitus. With regard to glycemic control, no clinically meaningful differences were observed between the two insulin analogs. Likewise, the risk of hypoglycemia was comparable with both basal insulin regimens. Notably, however, treatment with insulin detemir often required twice-daily insulin injections and a higher daily insulin dose (on average $0.26 \mathrm{U} /$ $\mathrm{kg}$ [95\% CI 0.11 to 0.41$]$ ) to achieve the same level of glycemic control as with insulin glargine, which may have cost implications. On the other hand, insulin detemir was associated with less weight gain (mean difference, $-0.91 \mathrm{~kg}$ [ $95 \%$ CI -1.2 to -0.61$]$ ). The number of injection site reactions was generally low, but slightly more frequent with insulin detemir (1.8\% of the patients) than with insulin glargine $(0.4 \%)$. Data regarding quality of life, health economics, and, most importantly, effects on diabetes-related morbidity and mortality could not be retrieved from the trials and will most certainly require larger studies with longer duration than those included in this meta-analysis.

\section{INSULIN DEGLUDEC: AN ULTRA LONG-ACTING BASAL INSULIN}

Insulin degludec, an ultra-long-acting basal insulin, versus insulin glargine in basal-bolus treatment with mealtime insulin aspart in type 1 diabetes (BEGIN Basal-Bolus Type 1): a phase 3, randomized, open-label, treat-to-target non-inferiority trial

Heller $S^{1}$, Buse $J^{2}$, Fisher $M^{3}$, Garg $S^{4}$, Marre $M^{5}$, Merker $L^{6}$, Renard $E^{7}$, Russel-Jones $D^{8}$, Philotheou $A^{9}$, Ocampo Francisco $A M^{10}$, Pei $H^{11}$, Bode $B^{12}$ on behalf of the BEGIN Basal-Bolus Type 1 Trial Investigators 
${ }^{1}$ University of Sheffield, Sheffield, United Kingdom; ${ }^{2}$ University of North Carolina, School of Medicine, Chapel Hill, NC; ${ }^{3}$ Glasgow Royal Infirmary, Glasgow, United Kingdom; ${ }^{4}$ Barbara Davis Center for Childhood Diabetes, Aurora, CO; ${ }^{5}$ Hôpital BichatClaude Bernard, Assistance Publique des Hôpitaux de Paris, Paris, France; ${ }^{6}$ Diabetes und Nierenzentrum, Dormagen, Germany; ${ }^{7}$ Montpellier University Hospital, Montpellier, France; ${ }^{8}$ Royal Surrey County Hospital, University of Surrey, Surrey, United Kingdom; ${ }^{9}$ University of Cape Town Private Academic Hospital, Cape Town, South Africa; ${ }^{10}$ Nowo Nordisk, Soeborg, Denmark; ${ }^{11}$ Novo Nordisk, Princeton; NJ; and ${ }^{12}$ Atlanta Diabetes Associates, Atlanta, GA

Lancet 2012; 379: 1489-1497

\section{Background}

To investigate the efficacy and safety of insulin degludec, administered once daily together with mealtime insulin aspart in patients with type 1 diabetes. Comparison was made with a similar basal-bolus regimen consisting of insulin glargine and aspart.

\section{Methods}

This was a multicenter (79 centers in six countries), openlabel, treat-to-target, non-inferiority trial consisting of 629 adult patients ( $\geq 18$ years) with type 1 diabetes on basal-bolus insulin therapy for at least one year and $\mathrm{HbA} 1 \mathrm{c} \leq 10 \%$ $(86 \mathrm{mmol} / \mathrm{mol})$. Subjects were randomly randomized $(3: 1$ ratio) to be treated for 52 weeks with once-daily subcutaneous injections of insulin degludec or insulin glargine. All patients received insulin aspart at mealtimes. The primary outcome was non-inferiority of degludec to glargine, in terms of decrease in $\mathrm{HbA1c}$ after 52 weeks (intention to treat analysis).

\section{Results}

At 52 weeks, the mean reduction of $\mathrm{HbA1c}$ was similar for the two basal insulin therapies; insulin degludec $-0.40 \pm 0.03 \%$ and insulin glargine $-0.39 \pm 0.0 .7 \%$ (estimated treatment difference $-0.01 \%$ [ $95 \% \mathrm{CI}-0.14$ to 0.11$] ; \mathrm{p}<0.0001$ for non-inferiority testing). Similarly, there was no difference in the relative number of patients achieving target $\mathrm{HbA1c}(<7 \%$ $[<53 \mathrm{mmol} / \mathrm{mol}]$ ) between the degludec and glargine groups ( $40 \%$ and $43 \%$, respectively). The total rate of confirmed hypoglycemia (plasma glucose $<3.1 \mathrm{mmol} / 1$ or severe hypoglycemic episodes) was comparable in the two treatment groups; 42.54 and 40.18 episodes per patient-year of exposure for insulin degludec and insulin glargine, respectively, giving an estimated degludec to glargine rate ratio of 1.07 ([0.89-1.28]; $\mathrm{p}=0.48$ ). The rate of nocturnal confirmed hypoglycemia was $25 \%$ lower in the degludec group than in the glargine group (4.41 and 5.86 episodes per patient-year of exposure, respectively); the estimated rate ratio being 0.75 ([0.59 - 0.96]; $\mathrm{p}=0.021)$. The overall rate of serious adverse events was comparable in the two treatment groups.

\section{Conclusions}

Insulin degludec therapy results in effective glycemic control with less risk of nocturnal hypoglycemia and might therefore be suitable for basal insulin supplementation in patients with type 1 diabetes.
Insulin degludec, an ultra-long-acting basal insulin, versus insulin glargine in basal-bosul treatment with meantime insulin aspart in type 2 diabetes (BEGIN Basal-Bolus Type 2): a phase 3, randomized, open-label, treat-to-target non-inferiority trial

Garber $A J^{1}$, King $A B^{2}$, Del Prato $S^{3}$, Sreenan $S^{4}$, Balci $M K^{5}$, Muños-Torres $M^{6}$, Rosenstock $J^{7}$, Endahl $L A^{8}$, Ocampo

Francisco $A M^{8}$, Hollander $P^{9}$ on behalf of the NN1250-3582 (BEGIN BB T2D) Trial Investigators

${ }^{1}$ Baylor College of Medicine, Houston, $\mathrm{TX}_{;}{ }^{2}$ Diabetes Care Center, Salinas, CA; ${ }^{3}$ University of Pisa, Pisa, Italy; ${ }^{4}$ Connolly Hospital, Dublin, Ireland; ${ }^{5}$ Akdeniz University School of Medicine, Antalya, Turkey; ${ }^{6}$ University Hospital San Cecilio, Granada, Spain; ${ }^{7}$ Dallas Diabetes and Endocrine Center at Medical City, Dallas, TX; ${ }^{8}$ Novo Nordisk, Soeborg, Denmark; and ${ }^{9}$ Baylor Medical Center, Dallas, TX

Lancet 2012; 379: 1498-1507

\section{Background}

To compare the efficacy and safety of insulin degludec and insulin glargine in patients with type 2 diabetes on basalbolus insulin therapy.

\section{Methods}

A multicenter (123 sites in 12 countries), open-label, treatto-target, non-inferiority trial. Adult patients with type 2 diabetes on any type of insulin regimen for at least three months (with or without oral hypoglycemic agents) and with $\mathrm{HbA} 1 \mathrm{c}$ between $7.0-10.0 \%$ were eligible. In total, 1,006 patients were included and randomly randomized (3:1 ratio) to be treated for 52 weeks with once-daily injections of insulin degludec $(n=755)$ or insulin glargine $(n=251)$ as basal insulin supplementation. Stratification was made according to type of previous insulin regimen. Basal insulin was titrated to achieve target plasma glucose between 3.9 and less than $5.0 \mathrm{mmol} / 1$ before breakfast (self-measured plasma glucose). The primary outcome was non-inferiority of degludec to glargine, assessed as change in $\mathrm{HbA1c}$ from baseline to week 52 (full analysis set). Rates of hypoglycemia were also assessed in all treated patients.

\section{Results}

The full analysis set included 744 patients randomized to insulin degludec and 248 patients allocated to insulin glargine. On average, they were $58.9 \pm 9.3$ years old, with diabetes duration of $13.5 \pm 7.3$ years. Their mean $\mathrm{HbA} 1 \mathrm{c}$ was $8.3 \pm 0.8 \%$, and their mean fasting plasma glucose was $9.2 \pm 3.1 \mathrm{mmol} / 1$. At 52 weeks, $\mathrm{HbA} 1 \mathrm{c}$ was reduced by $1.1 \%$ in the degludectreated patients and by $1.2 \%$ in the glargine-treated patients; the estimated treatment difference between the two groups being $0.08 \%$ ( $95 \% \mathrm{CI}-0.05$ to 0.21$)$, confirming non-inferiority. Total rates of confirmed hypoglycemia (plasma glucose $<3.1 \mathrm{mmol} / 1$ or severe hypoglycemic episodes) were lower in the degludec group than in the glargine group (11.1 and 13.6 episodes per patient-year of exposure, respectively), with an estimated rate ratio of 0.82 ([0.69-0.99]; $\mathrm{p}=0.0359$ ). Similarly, the rate of nocturnal confirmed episodes of hypoglycemia was lower with degludec than with glargine (1.4 vs. 1.8 episodes per patient-year of exposure; estimated rate ratio 0.75 
[0.58 - 0.99]; $p=0.0399)$. Rates of severe hypoglycemic episodes requiring assistance were low and similar in the two groups (0.06 and 0.05 episodes per patient-year of exposure for degludec and glargine, repectively). Likewise, rates of other types of adverse events were comparable in degludecand glargine-treated patients.

\section{Conclusions}

Basal insulin supplementation with insulin degludec results in lower rates of hypoglycemia than those with insulin glargine and might therefore be useful in patients with advanced type 2 diabetes to improve glycemic control with less risks of hypoglycemia and its associated hazards.

\section{Improved health status with insulin degludec compared with insulin glargine in people with type 1 diabetes}

Home $P D^{1}$, Meneghini $L F^{2}$, Wendisch $U^{3}$, Ratner $R E^{4}$, Johansen $T^{5}$, Christensen $T E^{5}$, Jendle $J^{6}$, Roberts $A P^{7}$, Birkeland $K I^{8}$

${ }^{1}$ Institute of Cellular Medicine-Diabetes, Newcastle University, Newcastle upon Tyne, United Kingdom; ${ }^{2}$ Miller School of Medicine, University of Miami, Miami, FL; ${ }^{3}$ Gemeinschaftspraxis für Innere Medizin und Diabetologie, Hamburg, Germany; ${ }^{4}$ Medstar Health Research Institute, Hyattsville, MD; ${ }^{5}$ Novo Nordisk A/S, Soeborg, Denmark; ${ }^{6}$ Endocrine and Diabetes Centre, Karlstad Hospital, Faculty of Health Sciences, Örebro University, Örebro, Sweden; ${ }^{7}$ Endocrine and Metabolic Unit, Royal Adelaide Hospital, Adelaide, SA, Australia; and ${ }^{8}$ Faculty of Medicine, University of Oslo, Department of Endocrinology, Oslo University Hospital, Oslo, Norway

Diabet Med 2012; 29: 716-720

\section{Background}

The effect of insulin degludec on health status was assessed and compared with insulin glargine in patients with type 1 diabetes.

\section{Methods}

Adult patients with type 1 diabetes were randomly randomized (open-label) to be treated for 16 weeks with oncedaily injections of either insulin degludec $(n=59)$ or insulin glargine $(n=59)$. All patients received insulin aspart at mealtimes. Health status was assessed with a validated questionnaire (Short Form 36 Health Survey [SF-36], version 2), consisting of eight domains that can be combined to provide two summary component scores for mental and physical well-being.

\section{Results}

At 16 weeks, the mean $\mathrm{HbA1c}$ reduction was comparable for the two insulin therapies. In comparison with glargine, the rate of confirmed nocturnal hypoglycemia $(<3.1 \mathrm{mmol} / \mathrm{l})$ was significantly reduced (relative rate 0.42 [ $95 \%$ CI 0.25 to 0.69$]$ ) and overall hypoglycemic events numerically less frequent (relative rate 0.72 [95\% CI 0.52 to 1.00]) in the degludec group. At the same time, the SF-36 summary component score for mental well-being showed a significant improvement in favor of the degludec therapy (+3.01 [95\% CI 0.32 to 5.70]). This was due to significant differences between the degludec vs. the glargine study groups in the social functioning $(+8.04$ [95\% CI
1.89 to 14.18$])$ and the mental health domains (+2.46 [95\% CI 0.10 to 4.82$]$ ). Calculation of Cohen's effect size for the mental component score (0.42) suggested a small-to-medium clinically meaningful difference. There were no significant differences between the study groups in the other six domains or in the combined physical component score.

\section{Conclusions}

While achieving similar overall glycemic control as glargine, degludec produced an improvement in mental wellbeing, as assessed with the SF-36 mental component score. This improvement, together with the causal improvements in the social functioning and mental health domains with degludec compared to glargine, may be associated with the concomitant reduction in hypoglycemic event rate.

\section{Comment}

As previously described, insulin degludec is a soluble, basal insulin analog with a smooth and stable pharmacokinetic profile; its duration of action exceeds $24 \mathrm{~h}$ and the within-subject variability is low. The ultra long-action profile is mainly the result of a slow and stable release of insulin degludec monomers from soluble multihexamers that form after subcutaneous administration. Last year, we reviewed the first published phase 2 , proof-of-concept trials in which the efficacy and safety of insulin degludec were compared with insulin glargine in patients with type 1 and type 2 diabetes mellitus. Overall, the findings indicated that basal insulin supplementation with insulin degludec resulted in principally the same glycemic control as that with insulin glargine, whereas the risk of hypoglycemia-and especially nocturnal hypoglycemiaseemed to be further reduced. In essence, these results were corroborated in the two new, 52-week, treat-to-target phase 3 studies, during which insulin degludec was compared with insulin glargine as basal insulin in a basalbolus insulin regimen in patients with type 1 and type 2 diabetes mellitus. Hence, while achieving almost identical improvements in glycemic control (HbA1c), treatment with insulin degludec significantly reduced the rate of nocturnal hypoglycemic events in both type 1 and type 2 diabetes; in the latter patients the rate of overall hypoglycemia was also significantly lower. With regard to weight gain or other adverse effects, there were no apparent differences between insulin degludec and insulin glargine regimens, neither in type 1 nor in type 2 diabetes.

Whether the reduced risk of hypoglycemia observed with insulin degludec in the phase 2 and 3 studies translates into improvements in health-related quality of life is still a matter of uncertainty. In the study by Home et al., health status was assessed using a validated generic questionnaire in one of the aforementioned phase 2 studies in patients with type 1 diabetes (4). Significant improvements were shown for insulin degludec against insulin glargine in two out of four domains of mental health assessments but none of the four domains evaluating physical well-being; the observed significant differences were considered to represent a small-tomoderate effect size from a clinical point of view. In contrast, in the larger phase 3 trial in type 1 diabetes, in 
which the same methodology for assessments of health status was used, no differences were found between insulin degludec- and insulin glargine-based regimens. Moreover, in the phase 3 trial in patients with type 2 diabetes, only one domain in the physical health status evaluations (bodily pain) was significantly improved with insulin degludec compared with insulin glargine, but otherwise no significant differences were recorded. Hopefully, more data from other long-term trials will clarify in more detail the potential benefits of this novel basal insulin analog, which probably will be available for clinical use in the near future.

\section{Pegylated Insulin Lispro: A New Approach to Basal Insulin Therapy}

An alternative approach to retard insulin absorption is poly(ethylene glycol) (PEG)ylation (5). When electrophilically activated derivatives of low-molecular-weight monomethoxyPEG (mPEG) are chemically coupled to insulin via its amino groups at positions phenylalanine-B1 or lysine-B29, an amide bond is formed between the polymer and protein. Apparently, the site-specific attachment of mPEG to insulin does not substantially alter the insulin's secondary/tertiary structure, self-association behavior, or potency in vivo. However, mPEG attachment does significantly enhance insulin's resistance to aggregation. A recent review by Bolli et al. (6) covers what is known so far about this insulin: A novel, controlled release formulation has been developed with PEGylated human insulin encapsulated in microspheres that produce multiday release in vivo. In animal studies, PEGinsulin microspheres administered subcutaneously as a single injection produced $<1 \%$ release of insulin in the first day, but then lowered the serum glucose levels of rats with diabetes to values $<200 \mathrm{mg} / \mathrm{dL}$ for approximately 9 days; when doses were given at 7-day intervals, steady-state drug levels were achieved after only two doses. Eli Lilly has described a longacting lispro insulin formulation that is PEGylated with highmolecular-weight PEG. It is highly soluble at physiological $\mathrm{pH}$, has an extended duration of action, and is characterized by $\mathrm{PK}, \mathrm{PD}$, and/or activity peak-trough ratios of less than two. Initial PD data in normal humans without diabetes indicate that PEGylated lispro has a flatter action profile compared with insulin glargine, along with prolonged activity (6).

\section{SHORT-ACTING INSULIN PREPARATIONS}

\section{U-100, pH-Neutral formulation of VIAject( $($ ): faster onset of action than insulin lispro in patients with type 1 diabetes}

Heinemann $L^{1}$, Nosek $L^{1}$, Flacke $F^{2}$, Albus $K^{2}$, Krasner $A^{2}$, Pichotta $P^{2}$, Heise $T^{1}$, Steiner $S^{2}$

${ }^{1}$ Profil Institut für Stoffwechselforschung, Neuss, Germany; and ${ }^{2}$ Biodel Inc., Danbury, CT

Diabetes Obes Metab 2012; 14: 222-227

\section{Background}

VIAject ${ }^{\circledR}$ is a formulation of human insulin with a very fast onset of action. Previous studies used VIAject in a concentra- tion of $25 \mathrm{U} / \mathrm{ml}$ and a $\mathrm{pH}$ of 4 (VIAject 25 [VJ25]). Objective of this double-blind, three-way crossover study was to compare the pharmacodynamic/pharmacokinetic properties of a novel formulation of VIAject with a concentration of $100 \mathrm{U} / \mathrm{ml}$ and a neutral pH (VIAject 7 [VJ7]) with VJ25 and insulin lispro (LIS).

\section{Methods}

Forty-three patients with type 1 diabetes (aged 43 [21-65] years, BMI 24.1 [20-28] $\mathrm{kg} / \mathrm{m}^{2}$, and $\mathrm{HbA1c} 7.5$ [5.7-9.5]\%) participated in this study. They received subcutaneous injections of $12 \mathrm{U}$ of each insulin formulation under euglycemic glucose clamp conditions.

\section{Results}

VJ7 was bioequivalent to VJ25 (90\% confidence interval [CI] of the ratios for total insulin AUCs and maximum insulin concentration [C(INS max)] was within 0.80 to 1.25$)$. VJ7 showed a faster absorption compared to LIS (time to C[INS $\max ] 23$ vs. $60 \mathrm{~min}$; difference [CI] $-30[-35$ to -23$])$ and faster onset of action (time to early half-maximal glucose infusion rate [GIR] 25 vs. $44 \mathrm{~min} ;-18$ [-26 to -10$]$ ), and a higher AUC of glucose infusion rate (AUC[GIR]) in the first $60 \mathrm{~min}$ after injection (176 vs. $107 \mathrm{mg} / \mathrm{kg}$; ratio 1.65 [1.27 to 2.14]), contributing to a slightly higher value for AUC(GIR 0-480) (1263 vs. $1095 \mathrm{mg} / \mathrm{kg} ; 1.15$ [1.06 to 1.26$])$. Maximum GIR was similar between VJ7 and LIS (6.1 vs. $6.6 \mathrm{mg} / \mathrm{kg} / \mathrm{min}$; ratio 0.93 [0.86 to 1.01]), whereas the duration of action (t[GIR50\%-late]) was longer with VJ7 (274 vs. $228 \mathrm{~min} ; 50$ [25 to 73]).

\section{Conclusions}

This formulation of VIAject is bioequivalent to the previously used formulation and has a faster absorption/onset of action than LIS.

\section{Improved postprandial glycemic control in patients with type 2 diabetes from subcutaneous injection of insulin lispro with hyaluronidase}

Hompesch $M^{1}$, Muchmore $D B^{2}$, Morrow $L^{1}$, Ludington $E^{2}$, Vaughn $D E^{2}$

${ }^{1}$ Profil Institute for Clinical Research, Chula Vista, CA; and ${ }^{2}$ Halozyme Therapeutics Inc, San Diego, CA

Diabetes Technol Ther 2012; 14: 218-224

\section{Background}

Previous studies in healthy subjects and patients with type 1 diabetes have shown that coinjection with hyaluronidase accelerates the absorption of insulin. In this study, the postprandial glucose response was investigated in patients with type 2 diabetes following administration of insulin lispro with or without recombinant human hyaluronidase ( $\mathrm{rHuPH} 20)$ and after coinjection of regular human insulin and rHuPH20.

\section{Methods}

Double-blind, three-way crossover study design, in which insulin pharmacokinetics and glucose responses to a standardized liquid meal ( $80 \mathrm{~g}$ of carbohydrates) were compared in patients with type 2 diabetes $(n=21)$ following subcutaneous injections of individually optimized doses of insulin 
lispro $\pm r H u P H 20$ and regular human insulin + rHuPH20. The optimum dose (targeting postprandial glucose excursion of $70-140 \mathrm{mg} / \mathrm{dl}$ ) of each preparation was individually assessed by a fixed-dose escalation procedure in three dose-finding meals.

\section{Results}

Coinjection of insulin lispro and $\mathrm{rHuPH} 20$ augmented the pharmacokinetics in comparison to administration of lispro alone (time to peak insulin concentration 43 vs. $74 \mathrm{~min}$, $\mathrm{p}=0.0045)$, with higher insulin levels in the first $60 \mathrm{~min}(184 \%$ of control, $p=0.0001)$ and lower levels after $120 \mathrm{~min}(67 \%$ of control, $p=0.0001)$. This was mirrored by improvements in both total hyperglycemic excursions (area under the curve 0-4 hours $>140 \mathrm{mg} / \mathrm{dl}, 56 \%$ of control; $\mathrm{p}=0.048$ ) and hypoglycemic excursions (area under the curve for 0-8 hours $<70$ $\mathrm{mg} / \mathrm{dl}, 34 \%$ of control; $\mathrm{p}=0.033)$. On average, the optimum dose of insulin lispro was reduced by $8 \%$ when coinjected with $\mathrm{rHuPH} 20$ (from $0.275 \mathrm{U} / \mathrm{kg}$ to $0.254 \mathrm{U} / \mathrm{kg} ; \mathrm{p}=0.04$ ). Coinjection of regular human insulin together with $\mathrm{rHuPH} 20$ resulted in glucose responses and optimum doses similar to those with insulin lispro alone. All preparations were well tolerated.

\section{Conclusions}

Coinjection of insulin lispro and $\mathrm{rHuPH} 20$ were associated with improved postprandial glucose control compared with insulin lispro alone, with lower insulin requirements and reduced risk of hypoglycemia.

\section{Comment}

We have reported on the VIAject (Linjeta) human insulin preparation in previous years and described the regulatory issues. In the past year, two presentations at the ADA scientific sessions (7) reported progress regarding a new formulation and pain associated with injecting Linjeta. Concentrated formulations of Biodel's ultra rapid-acting insulin may be absorbed more rapidly than existing concentrated insulin formulations, as demonstrated in the above paper of Heinemann et al., and may therefore become an important component of an effective step in the development of an artificial pancreas. Currently, regular insulin at concentrations of 500 units per milliliter is used to treat extremely insulin-resistant patients with diabetes. While these formulations are five times more concentrated than typical insulin presentations, they have a prolonged duration of action that may be undesirable for use with an artificial pancreas. Biodel Inc. announced that the Small Business Innovation Research program of the National Institutes of Health (NIH) has awarded them a grant for the development of concentrated ultra rapid-acting insulin formulations for use in an artificial pancreas. VIAject at high concentrations may be suited to provide sufficient quantities of insulin in an external artificial pancreas pump device that has a limited volume capacity. We will continue to monitor this development.

Last year, we also reported on the ongoing development of coadministration of insulin and hyaluronidase to facilitate the absorption of insulin and the mechanisms underlying this phenomenon. In short, hyaluronidase mediates a depolymerization of the hyaluronan matrix in the subcutaneous tissue space, which results in a dilution effect that favors dissociation of insulin hexamers and, hence, enhances the rate of insulin absorption. In addition, the insulin molecules are spread over a larger capillary bed, which facilitates their absorption. As reviewed, previous experimental studies in healthy subjects and patients with type 1 diabetes mellitus have demonstrated that coadministration of recombinant human hyaluronidase speeds up the absorption of both regular human insulin and insulin lispro, leading to faster insulin exposure and a corresponding improvement in the postprandial glycemic control. The present study by Hompesch et al. demonstrates that the same is also true in patients with type 2 diabetes. Clearly, however, clinical trials with longer time-use of coadministration of insulin and recombinant human hyaluronidase are needed to evaluate its clinical efficacy and safety issues associated with chronic exposure to hyaluronidase.

\section{Author Disclosure Statement}

T.D. has received honoraria for speaking engagements from several companies involved in the diabetes field. Furthermore he has received grant support from these companies (Abbott, Sanofi, Bayer, Boehringer, Roche, Johnson \& Johnson, Lilly, Medtronic, Menarini, DexCom, and NovoNordisk) for the conduct of studies or scientific meetings. J.B. has received consulting fees as a member of scientific advisory boards from Abbott Diabetes Care, AstraZeneca, Medtronic, MSD, and Sanofi-Aventis; and honoraria for lectures from Sanofi-Aventis and NovoNordisk.

\section{References}

1. Pickup, JC. Insulin pumps. In: Phillip M, Battelino T (eds.), ATTD Year Book 2012, 51-52.

2. Jovanovič, L. Letter written in response to van Bon et al.: "Insulin glulisine compared to insulin aspart and to insulin lispro administered by continuous subcutaneous insulin infusion in patients with type 1 diabetes: a randomized controlled trial. Diabetes Technol Ther 2011; 13: 869-870.

3. Heinemann, L. Biosimilar insulins. Expert Opin Biol Ther 2012; 12: 1009-16.

4. Birkeland, KI, Home, PD, Wendisch, U, Ratner, RE, Johansen, T, Endahl, LA, Lyby, K, Jendle, JH, Roberts, AP, DeVries, JH, Meneghini, LF. Insulin Degludec in type 1 diabetes: a randomized controlled trial of a new-generation ultra-longacting insulin compared with insulin glargine. Diabetes Care 2011; 34: 661-665.

5. Beals, JM, Cutler, GB, Doyle, BL, Hansen, RJ, Li, S, Shirani, S, Zhang, L. Pegylated insulin Lispro compounds (Publication Date: 05/05/2011). http://freepatentsonline.com/y2011/ 0105392.html (accessed Nov. 12, 2012)

6. Bolli, GB, Andreoli, AM, Lucidi, P. Optimizing the replacement of basal insulin in type 1 diabetes mellitus: no longer an elusive goal in the post-nph era. Diabetes Technol Ther 2011; 13, Suppl. 1: S43-53.

7. Pohl, R, Hauser, R, Wilson, BR, Li, M, Seibert, R, Reddy, P, Guinness, M, Jackson, M, DeSouza, E. 920-Pharmacokinetic 
and Pharmacodynamic Comparison of New Ultra-RapidActing Insulin Formations in Diabetic Miniature Swine and Castle, JR, Morrow, L, Pitts, A, El Youssef, J, Blanchfield, L, Pichotta, P, Hompesch, M, Ward, WK, Krasner, A, 350-OREvaluations of Modified Ultra-Rapid-Acting Linjeta TM Formulations BIOD-105 and BIOD-107 in Patients with Type 1 Diabetes. Both papers presented at the $72^{\text {nd }}$ Scientific Sessions of the American Diabetes Association June 8-12, 2012, Philadelphia, PA. Pharmacokinetic and Pharmacodynamic Com- parison of New Ultra-Rapid-Acting Insulin Formulations in Diabetic Miniature Swine "\#920-P, Poster Category 01-B/C Clinical Therapeutics/New Technology - Insulin Delivery Systems and \#350-OR, Oral Session on Insulin Analogs Evaluations of Modified Ultra-Rapid-Acting Linjeta ${ }^{\mathrm{TM}}$ Formulations BIOD-105 and BIOD-107 in Patients with Type1 Diabetes"

8. The ORIGIN Trial Investigators basal insulin and cardiovascular and other outcomes in dysglycemia. $N$ England J of Med 2012; 367: 319-328. 